\title{
Estimulación en el hogar y desarrollo motor en niños mexicanos de 36 meses
}

\author{
Erika Osorio, M en Psic,, (I) Luisa Torres-Sánchez, D en SP,(2) María del Carmen Hernández, Psic, ${ }^{(1)}$ \\ Lizbeth López-Carrillo, PhD, (2) Lourdes Schnaas, M en Psic.(I)
}

\section{Osorio E,Torres-Sánchez L, Hernández MC, López-Carrillo L, Schnaas L. \\ Estimulación en el hogar y desarrollo motor en niños mexicanos de 36 meses. Salud Publica Mex 2010;52:14-22.}

\section{Resumen}

Objetivo. Identificar la relación entre la estimulación en el hogar y el desarrollo motor en niños de 36 meses de edad. Material y métodos. Se evaluó el desarrollo motor grueso y fino de 169 infantes (50.9\% varones) mediante la Escala de Desarrollo Motor de Peabody y se determinó la calidad de estimulación en el hogar en una evaluación previa con la Escala HOME a los 30 meses de edad. Resultados. La estimulación total en el hogar se asoció significativamente con un mejor desempeño en las áreas motora gruesa y fina. Aspectos particulares de esta estimulación se relacionaron con una mejor motricidad gruesa y fina. Conclusión. Equilibrio y locomoción en la motricidad gruesa y agarre e integración visomotora en la motricidad fina son los componentes motores asociados a aspectos particulares de la estimulación como la interacción madre-hijo, el reforzamiento de acciones positivas en forma verbal y la presencia de límites claros.

Palabras clave: niños; cuidadores; desarrollo; motricidad; México
Osorio E,Torres-Sánchez L, Hernández MC, López-Carrillo L, Schnaas L.

Stimulation at home and motor development among 36 month-old Mexican children.

Salud Publica Mex 2010;52:14-22.

\section{Abstract}

Objective. To identify the relationship between stimulation at home and motor development among 36 month-old children. Materials and Methods. The development of gross and fine motor skills of 169 infants $(50.9 \%$ boys and $49.1 \%$ girls) was assessed at the age of 36 months with the Peabody Developmental Motor Scale.The quality of home stimulation was determined during a prior evaluation (at 30 months) by means of the HOME Scale. Results. Total stimulation at home was significantly associated with better performance in the gross and fine motor areas. Particular aspects of this home stimulation were related to better gross and fine motor functions. Conclusion. Static balance and locomotion (gross motor skills) and grasping and visual-motor integration (fine motor skills) are associated with particular aspects of home stimulation, such as parent-child interaction, verbal reinforcement of the child's positive actions and providing the child with clear boundaries.

Key words: children; caregivers; development; motricity; Mexico

(I) Instituto Nacional de Perinatología Isidro Espinosa de los Reyes. México, DF, México.

(2) Instituto Nacional de Salud Pública. Cuernavaca, Morelos, México.

Fecha de recibido: 26 de junio de 2009 - Fecha de aceptado: 14 de octubre de 2009 Solicitud de sobretiros: Mtra. Lourdes Schnaas. Instituto Nacional de Perinatología Isidro Espinosa de los Reyes. Montes Urales 800, col. Lomas de Virreyes. I 1000 México DF, México.

E-mail:Ischnaas@hotmail.com 
E desarrollo infantil en los primeros años de vida es un tema que se ha estudiado continuamente por ser uno de los periodos críticos del ser humano que marca las bases para las siguientes etapas y que está influenciado por aspectos biológicos y ambientales.

La estimulación que los padres otorgan al niño en el hogar influye en su desarrollo mental y motor durante los primeros años antes de que el niño ingrese a la escuela. ${ }^{1-5}$ La motricidad se favorece cuando se incrementa la interacción materno-infantil, se combina la estancia del niño tanto en casa como en centros de cuidado infantil, ${ }^{6}$ se le da la oportunidad al niño de socializar en los primeros años con personas que no pertenecen a la familia, se le otorgan espacios suficientes para moverse libremente sin que el adulto lo mantenga cargado o permanezca en la cuna, y se le proporcionan juguetes adecuados a su edad que promuevan el desarrollo de sus habilidades. ${ }^{7,8}$

La madre interviene directamente en la estimulación de la motricidad, ayudando o inhibiéndola; madres jóvenes y con mejor nivel educativo se involucran más activamente en el juego del niño mejorando sus movimientos, ${ }^{9}$ mientras que madres con algún trastorno como la depresión disminuyen su sensibilidad y habilidad para proveer juegos que ayuden al niño a explorar su medio ambiente. ${ }^{1}$ Asimismo, la composición de la familia juega un papel importante; aquellas familias compuestas por mamá, papá, hijo(s), abuelos, tíos y primos otorgan mayor variedad en los juegos y permiten una libre exploración, aunque también pueden llegar a ser desfavorables por la poca organización temporal y física del ambiente, lo que introduce al niño en una sobreestimulación y sobrecarga la capacidad de éste para clasificar y responder al medio en que se desenvuelve. ${ }^{10}$

Para evaluar la calidad de la estimulación en el hogar algunos autores han utilizado cuestionarios que indagan acerca de las rutinas diarias del niño, ${ }^{4,6,7}$ mientras que otros han utilizado la prueba Stim ${ }^{11}$ (que mide la estimulación cognitiva en el hogar identificando la cantidad y calidad de material de juego y actividades de lectura padre-hijo) y la escala HOME para infantes (Home Observation for Measurement of the Environment). ${ }^{1-3,10}$ Esta última prueba es de particular interés porque los reactivos se agrupan en seis subescalas y, a diferencia de otras pruebas, se realiza dentro del hogar, calificando aspectos no sólo de las rutinas diarias del niño y el material de juego que tiene en casa para la estimulación cognitiva, sino también aquéllos de organización física de la casa, aceptación del niño en la familia, participación activa de los padres en el aprendizaje del niño, interacción madre-hijo y variedad de oportunidades en la estimulación diaria.
La escala HOME se ha utilizado en diversos estudios relacionados con el desarrollo infantil para obtener un indicador de la estimulación global en el hogar, ${ }^{1-3}$ asociado al desarrollo mental y motor en los primeros años de vida del niño, ${ }^{12}$ lo cual ha sido de utilidad para desarrollar estrategias de intervención familiar. En México, $83.8 \%$ de los niños y $84.4 \%$ de las niñas menores de seis años permanecen al cuidado de sus madres en casa. ${ }^{13}$ Sólo dos estudios en el país evalúan el papel de la estimulación en el hogar sobre el desarrollo motor infantil; uno de ellos realizado en una zona de bajo nivel socioeconómico del Distrito Federal en el que se evaluó el desarrollo psicomotor mediante el Perfil de Conductas del Desarrollo, y que no reporta asociación entre la estimulación en el hogar y desarrollo motor, aunque carece de un control adecuado de confusores. ${ }^{10} \mathrm{El}$ otro realizado en población rural, donde se usó la escala de Gesell para evaluar el desarrollo motor grueso, encontró que el abandono temprano del gateo y correr a temprana edad se asocia con una mayor estimulación en el hogar. ${ }^{14}$ Sin embargo, no se cuenta con estudios que asocien aspectos particulares de la estimulación en el hogar con la motricidad infantil fina y gruesa. El presente trabajo está encaminado a identificar la relación entre algunos factores de la estimulación en el hogar evaluados con la escala HOME y aspectos particulares del desarrollo motor evaluados con la Escala de Desarrollo Motor de Peabody en niños de escasos recursos en el estado de Morelos.

\section{Material y métodos}

De enero de 2001 a junio de 2005 se conformó una cohorte de mujeres en edad reproductiva, sin antecedentes de enfermedades crónicas, ni en tratamiento con anticonvulsivos (condición necesaria por la medición del ácido fólico que se llevó a cabo en estas mujeres, pues estos medicamentos modifican el metabolismo del mismo) y que residían en cuatro municipios del estado de Morelos, México. El objetivo principal del estudio fue evaluar el impacto de la exposición prenatal a compuestos organoclorados sobre el neurodesarrollo infantil. Previamente fueron reportados detalles sobre la conformación de la cohorte y la evaluación del neurodesarrollo durante el primer año de vida de 244 niños. ${ }^{15}$ Brevemente, el seguimiento de las participantes se inició inmediatamente después del matrimonio civil y continuó efectuándose antes, durante y después del embarazo. Durante cada una de estas etapas y mediante la aplicación de cuestionarios estructurados, se obtuvo información acerca de las características sociodemográficas, antropométricas y de salud de la madre, así como evolución del embarazo y características del nacimiento. Las visitas postnatales 
iniciaron al mes de edad y continuaron cada 6 meses hasta los 5 años. Durante cada una de estas visitas se obtuvo información acerca de la antropometría, tipo de alimentación, estado de salud de los niños y neurodesarrollo infantil. Este reporte sólo incluye información acerca del desarrollo motor de 169 niños a quienes se les aplicó la Escala de Desarrollo Motor de Peabody ${ }^{16}$ a los 36 meses de edad. La estimulación en el hogar medida a través de la escala de $\mathrm{HOME}^{17}$ fue evaluada a los 6 y 30 meses de edad. El cociente intelectual de la madre se evaluó mediante la aplicación de una prueba específica.

Al momento del ingreso al estudio, se obtuvo una carta de consentimiento informado y una de re-consentimiento para el seguimiento de los niños de cada una de las participantes. Este estudio contó con la aprobación de los Comités de Ética del Instituto Nacional de Salud Pública de México y del Instituto Nacional de Perinatología Isidro Espinosa de los Reyes.

\section{Evaluación del desarrollo motor}

El desarrollo motor de los niños se evaluó mediante la aplicación de una versión traducida al español de la Escala de Desarrollo Motor de Peabody Segunda Edición(PDMS-2) ${ }^{16}$ Esta escala está diseñada para evaluar a niños desde el nacimiento hasta los 5 años de edad y permite medir habilidades motoras interrelacionadas que se desarrollan a temprana edad, a través de seis subpruebas: reflejos, equilibrio estático, locomoción, manipulación de objetos, agarre e integración viso-motora. Se aplica de forma individual pidiéndole al niño que realice una serie de actividades motoras; cada uno de los reactivos se califica con criterios específicos otorgándoles una calificación de 2, 1 y 0: 2 cuando la conducta motora está completamente establecida, 1 cuando la conducta motora todavía se está desarrollando y no está completamente establecida y 0 cuando está ausente. Los resultados de las seis subpruebas se usan para generar tres índices globales de desempeño motor: El Índice Motor Grueso (IMG), compuesto por reflejos, equilibrio, locomoción y manipulación de objetos; el Índice Motor Fino (IMF), compuesto por agarre e integración visomotora y, finalmente, el Índice Motor Total (IMT) que se obtiene a partir de los índices descritos previamente. Esta escala no está validada en México; sin embargo, ha sido utilizada en muchos otros estudios en diferentes países. ${ }^{2,18-22}$ La mayoría de las puntuaciones obtenidas por los niños en este estudio se encontraron dentro de los límites promedio de la escala.

\section{Evaluación de la calidad del ambiente en el hogar}

A los 6 y 30 meses de edad se aplicó una versión traducida al español de la escala HOME para infantes. ${ }^{17}$ Este instrumento está diseñado para medir la calidad del ambiente familiar en el hogar en niños de 0 a 3 años y cubre los siguientes aspectos: interacción padres-hijos, el tipo de juguetes disponibles, calidad de estimulación emocional, cognitiva y social, así como la organización física que prevalece en el hogar. A esta prueba se le asignó un puntaje crudo de acuerdo con la sumatoria de las seis subescalas que la conforman: respuesta emocional y verbal de la madre, abstinencia de restricción y castigo, organización del medio ambiente físico y temporal, provisión de material de juego apropiado, involucramiento materno con el niño y oportunidades de variación en la estimulación diaria. El puntaje varía entre 0 y 45 puntos.

La escala HOME que se aplica de 0 a 3 años consta de 45 reactivos contenidos en seis subescalas, que combinan técnicas de observación y entrevista para realizar la valoración. En el rango de la prueba (0.45), una mayor puntuación corresponde a una mejor calidad de la estimulación. Se administra en el hogar del niño estando éste presente y despierto. El sujeto de la entrevista es el cuidador principal (comúnmente la madre). La escala cataloga una variedad de interacciones, eventos y objetos del ambiente familiar del niño.

Para fines de esta investigación no se llevó a cabo un estudio de validación; sin embargo, para la versión original de la prueba se reporta una moderada a alta estabilidad y una confiabilidad que varía desde 0.64 para desarrollo mental y estimulación vocal a 0.89 para aspectos del ambiente físico.

\section{Cociente intelectual de la madre}

El cociente intelectual de la madre se evaluó durante alguna de las visitas postnatales, mediante una versión traducida al español y utilizada anteriormente de la prueba reducida de WAIS (Weschler Adults Intelligence Scale), ${ }^{23}$ que consiste en la aplicación de las subescalas de información, comprensión, semejanzas y cubos. La aplicación de todas las pruebas estuvo a cargo de dos psicólogas con experiencia en la aplicación de las mismas. 


\section{Análisis estadístico}

De acuerdo con el sexo del infante, se describieron características seleccionadas de la madre, el niño y la familia. El efecto de la calidad de la estimulación en el hogar sobre el desarrollo motor del niño a los 36 meses de edad se evaluó mediante modelos de regresión lineal. Para cada uno de los componentes (fino y grueso) se corrió un modelo de regresión lineal que consideró como potenciales confusores la edad (años), escolaridad (años), cociente intelectual y ocupación materna, peso al nacimiento $(\mathrm{g})$, sexo del recién nacido, antecedente de lactancia materna, orden al nacimiento, antropometría a los 36 meses y tipo de familia (nuclear y extensa).

En el modelo final sólo permanecieron aquellas variables que modificaron en más de $10 \%$ el coeficiente $\beta$ del modelo crudo. Para evaluar una posible modificación del efecto de la calidad de la estimulación en el hogar por el sexo del infante, se incluyó en el modelo un término de interacción entre ambas variables y se consideró como significativo un valor de $p<0.05$.

El diagnóstico del modelo consistió en la estimación de los residuos. El supuesto de normalidad de los residuos se evaluó mediante las pruebas de Shapiro-Wilks, así como la evaluación mediante histogramas y gráficas de qnorm. El análisis se realizó con el paquete estadístico STATA versión 9.2

\section{Resultados}

Al comparar las características maternas de acuerdo con el sexo del infante, no se observaron diferencias significativas entre las madres de niños y niñas, excepto en la escolaridad, donde las madres de los niños tuvieron en promedio más años de estudios (11.0 vs. 10.2 años). En relación con las características del infante y de la familia, los niños se caracterizaron por haber recibido menos lactancia y tener una circunferencia cefálica al momento de la evaluación significativamente mayor que las niñas, y una proporción marginalmente mayor de ellos pertenecía a una familia nuclear (48.8 vs. $34.9 \%)$ (cuadro I).

La evaluación de la motricidad a través de la Escala de Desarrollo Motor de Peabody (cuadro II) mostró que los niños presentaron un desempeño significativamente mejor que las niñas en el área de la locomoción y la manipulación de objetos, así como en la motricidad gruesa en general. En contraste, la motricidad fina, especialmente en relación con el agarre, fue significativamente mejor entre las niñas en comparación con los niños. Al comparar la estimulación en el hogar que reciben niños y niñas, se observó que en general los niños reciben mayor estimulación que las niñas (cuadro III),
Cuadro I

Características generales de la población de estudio. Morelos, MÉXICo, 2004-2008

\begin{tabular}{|c|c|c|c|}
\hline Características & $\begin{array}{l}\text { Niños } \\
(n=86)\end{array}$ & $\begin{array}{l}\text { Niñas } \\
(n=83)\end{array}$ & Valor "p" \\
\hline \multicolumn{4}{|l|}{ Madre } \\
\hline \multicolumn{4}{|l|}{ Edad (años) } \\
\hline Media $\pm \mathrm{DE}$ & $21.8 \pm 4.2$ & $22.0 \pm 3.9$ & 0.63 \\
\hline Mín-máx & $16-38$ & $|6-3|$ & \\
\hline \multicolumn{4}{|l|}{ Escolaridad (años) } \\
\hline Media $\pm \mathrm{DE}$ & $11.0 \pm 2.9$ & $10.2 \pm 2.9$ & 0.03 \\
\hline Mín-máx & $5-19$ & $5-20$ & \\
\hline \multicolumn{4}{|l|}{ Coeficiente intelectual } \\
\hline Media $\pm \mathrm{DE}$ & $87.3 \pm 11.7$ & $86.4 \pm 12.1$ & 0.32 \\
\hline Mín-máx & $61-123$ & $57-121$ & \\
\hline
\end{tabular}

Tipo de parto (\%)

$\begin{array}{llll}\text { Cesárea } & 57.0 & 54.2 .0 & 0.72\end{array}$

Infante

Peso al nacer $(\mathrm{kg})$

\begin{tabular}{lccc} 
Media \pm DE & $3.3 \pm 0.4$ & $3.2 \pm 0.48$ & 0.17 \\
\hline Mín-máx & $2.4-4 . I$ & $2.05-4.8$ &
\end{tabular}

Lactancia (\%)

\begin{tabular}{lccc} 
No & 14.0 & 3.6 & 0.04 \\
\hline$\leq 12$ semanas & 22.1 & 18.1 & \\
\hline$>12$ semanas & 63.9 & 78.3
\end{tabular}

Lugar que ocupa el niño

$\begin{array}{llll}\text { Primero } & 90.7 & 83.1 & 0.14\end{array}$

Segundo o más

$9.3 \quad 16.9$

Peso a la evaluación $(\mathrm{g}) *$

$\begin{array}{llll}\text { Media } \pm D E & 15.0 \pm 2.2 & 14.8 \pm 3.0 & 0.30\end{array}$

$9.6-23.0 \quad 11.0-26.5$

Talla a la evaluación $(\mathrm{cm}) *$

\begin{tabular}{lccc} 
Media $\pm \mathrm{DE}$ & $95.7 \pm 4.3$ & $95.6 \pm 4.7$ & 0.42 \\
\hline Min-máx & $85-105$ & $78-110$ &
\end{tabular}

Circunferencia cefálica $(\mathrm{cm})^{*}$

Media \pm DE $\quad 49.4 \pm 1.6 \quad 48.8 \pm 1.5$

Mín-máx $\quad 47-54 \quad 45-52 \quad 0.006$

Familia

Tipo de familia (\%)

\begin{tabular}{llll} 
Nuclear & 48.8 & 34.9 & \\
\hline Extensa & 51.2 & 65.1 & 0.07
\end{tabular}

*A los 36 meses de edad 


\section{Cuadro II}

Escala de desarrollo motor de Peabody* SEgún SEXo del INFANTE. Morelos, MéXico, 2004-2008

$\begin{array}{cccc}\text { Peabody } & \text { Niños } & \text { Niñas } & \text { Valor "p" }\end{array}$

\begin{tabular}{lccc}
$\begin{array}{l}\text { Motricidad gruesa } \\
\text { Locomoción } \\
\text { Media } \pm \text { DE }\end{array}$ & $9.0 \pm 1.1$ & $8.7 \pm 0.96$ & 0.03 \\
\hline $\begin{array}{c}\text { Mín-máx } \\
\text { Equilibrio } \\
\text { Media } \pm \text { DE }\end{array}$ & $6-12$ & $6-10$ & \\
\hline $\begin{array}{c}\text { Min-máx } \\
\text { Manipulación de objetos } \\
\text { Media } \pm \text { DE }\end{array}$ & $10.4 \pm 2.0$ & $10.7 \pm 1.8$ & 0.81 \\
\hline Mín-máx & $6-16$ & $6-15$ & \\
& $7-13 \pm 1.4$ & $9.0 \pm 1.3$ & $<0.001$ \\
$\begin{array}{c}\text { Motricidad fina } \\
\text { Agarre } \\
\text { Media } \pm \text { DE }\end{array}$ & $6-12$ & \\
\hline $\begin{array}{l}\text { Mín-máx } \\
\text { Integración visomotora }\end{array}$ & $9.8 \pm 2.4$ & $11.3 \pm 2.8$ & 0.000 \\
\hline Media \pm DE & $6-16$ & $6-17$ & \\
\hline Mín-máx & $9.6 \pm 1.2$ & $9.4 \pm 0.92$ & 0.13 \\
\hline
\end{tabular}

*A los 36 meses de edad y resaltan como marginalmente significativos el mayor involucramiento materno y mayores oportunidades de variación en la estimulación diaria observada entre los niños en comparación con las niñas $(p=0.06)$.

El efecto de la estimulación que reciben los niños en el hogar (Escala HOME para infantes) sobre la motricidad gruesa y fina se describe en el cuadro IV. Independientemente del sexo y la escolaridad materna, el desempeño en la motricidad gruesa se incrementa significativamente conforme aumenta la estimulación total recibida en el hogar. La respuesta emocional y verbal de la madre y la abstinencia de restricción y castigo son los aspectos de la estimulación en el hogar que influyen de manera significativa. Cercanamente a lo observado con la motricidad gruesa, el desempeño motor fino incrementa significativamente conforme aumenta la estimulación total recibida en el hogar, así como la respuesta emocional y verbal de la madre; no obstante, es evidente que una mayor cantidad de material de juego disponible y un mayor involucramiento materno influyen de manera significativa en el mejor desempeño motor observado en el área motora fina, independientemente del sexo del infante, la escolaridad materna, el tipo de familia (nuclear o extensa) y el lugar que ocupa el niño en el hogar.

\section{Cuadro III}

Escala HOME según sexo del infante. Morelos, México, 2004-2008

Características de la prueba

Escala HOME

Respuesta emocional y verbal de la madre Media \pm DE Mín-máx
Niños

$4-11$

$6.2 \pm 1.0$

$2-8$

$5.3 \pm 0.8$

$3-6$

$5.0 \pm 2.0$

I-9

$4.6 \pm 2.0$

$1-9$

Niñas
$(n=83)$

$8.7 \pm 1.8$

$4-11$

$6.1 \pm 0.7$

4-7

0.11

Organización del medio ambiente físico y temporal
Media $\pm \mathrm{DE}$

Mín-máx

Provisión de material de juego apropiado

Media $\pm \mathrm{DE}$

$3.7 \pm 1.5$

$3.3 \pm 1.5$

Media \pm DE

$0-6$

$2.7 \pm 1.0$

Media \pm DE

Mín-máx

$\frac{2.7 \pm 1.0}{0-5}$

$2.5 \pm 1$. $0-5$
$5.2 \pm 0.9$

$2-6$

Valor "p"

Puntuación Total

Media $+D E$

Mín-máx

$32.0 \pm 5.3$

$17-43$

$30.4 \pm 5.1$

17-42

salud pública de méxico / vol. 52, no. 1, enero-febrero de 2010 
Al analizar cada uno de los componentes de la motricidad gruesa, se observa que el efecto positivo de la estimulación total en el hogar y de la respuesta emocional y verbal de la madre es principalmente significativo sobre la locomoción, mientras que la abstinencia de restricción y castigo sólo incrementa significativamente el desempeño relacionado con el equilibrio estático. No se observó efecto significativo de la estimulación total en el hogar o de alguno de sus componentes sobre la manipulación de objetos. En cuanto a los aspectos de la motricidad fina evaluados, la estimulación total en el hogar mejora de manera significativa el agarre y la integración visomotora; lo mismo ocurre con la respuesta emocional y verbal de la madre y con el involucramiento materno. En el caso de los materiales de juego, una mayor provisión de juguetes apropiados sólo incrementa significativamente los aspectos relacionados con la integración visomotora (cuadro V).

\section{Cuadro IV}

Efecto de la escala HOME y sus componentes sobre la motricidad gruesa y fina. Morelos, México, 2004-2008

Estimulación en el hogar (escala HOME)

\begin{tabular}{lllll} 
Respuesta emocional y verbal de la madre & 0.67 & $0.06 ; 1.28^{\S}$ & 1.4 & $0.6 ; 2.14^{\S}$ \\
\hline Abstinencia de restricción y castigo & 1.30 & $0.06 ; 2.52^{\S}$ & 1.61 & $-0.05 ; 3.30$ \\
\hline Organización del medio ambiente físico y temporal & 0.53 & $-0.80 ; 1.85$ & 0.44 & $-1.33 ; 2.21$ \\
\hline Material de juego & 0.36 & $-0.24 ; 0.96$ & 0.90 & $0.13 ; 1.66^{\S}$ \\
\hline Involucramiento materno & 0.50 & $-0.22 ; 1.21$ & 1.84 & $0.96 ; 2.72^{\S}$ \\
\hline Variedad de estimulación diaria & -0.12 & $-1.15 ; 0.9 \mid$ & -0.42 & $-1.81 ; 0.97$ \\
\hline Total & 0.25 & $0.02 ; 0.48^{\S}$ & 0.57 & $0.28 ; 0.86^{\S}$
\end{tabular}

* Ajustado por: sexo y escolaridad materna (años)

‡ Ajustado por: sexo del infante, escolaridad materna (años), tipo de familia (nuclear / extendida) y lugar que ocupa el infante dentro de la familia (primero vs. segundo o más)

$\S p<0.05$

\section{CuadroV \\ EfEcto de LA ESCALA HOME y SUS COMPONENTES SOBRE LOS COMPONENTES DE LA MOTRICIDAD GRUESA Y FINA. Morelos, MÉxico, 2004-2008}

Estimulación en el hogar (escala HOME)

\begin{tabular}{|c|c|c|c|c|c|}
\hline \multicolumn{3}{|c|}{ Motricidad gruesa } & \multicolumn{3}{|c|}{ Motricidad fina } \\
\hline Locomoción* & Equilibrio & Manipulación de objetos ${ }^{\S}$ & & & Integración visomotora \\
\hline $95 \%$ IC & $95 \%$ IC & $95 \%$ IC & $\beta$ & $95 \%$ IC & $95 \%$ IC \\
\hline
\end{tabular}

Respuesta emocional y verbal de la madre

$\begin{array}{llllll}0.13 & 0.04 ; 0.2^{\&} & 0.14 & -0.02 ; 0.30 & 0.04 & -0.08 ; 0.20\end{array}$

$0.27 \quad 0.05 ; 0.50^{\&} \quad 0.17 \quad 0.08 ; 0.26^{\&}$ y castigo

$\begin{array}{llllllllll}0.10 & -0.9 ; 0.3 & 0.40 & 0.06 ; 0.72^{\&} & -0.004 & -0.25 ; 0.25 & 0.25 & -0.21 ; 0.71 & 0.15 & -0.04 ; 0.34\end{array}$

Material de juego

nvolucramiento mater

$\begin{array}{llllllllll}0.06 & -0.02 ; 0.14 & 0.05 & -0.12 ; 0.21 & 0.01 & -0.10 ; 0.13 & 0.93 & -0.13 ; 0.32 & 0.17 & 0.09 ; 0.26^{\&} \\ 0.06 & -0.04 ; 0.20 & 0.14 & -0.06 ; 0.33 & 0.04 & -0.10 ; 0.18 & 0.52 & 0.27 ; 0.78^{\&} & 0.14 & 0.05 ; 0.25^{\&} \\ 0.04 & 0.01 ; 0.08^{\&} & 0.05 & -0.006 ; 0.11 & 0.006 & -0.04 ; 0.05 & 0.11 & 0.03 ; 0.19^{\&} & 0.07 & 0.04 ; 0.10^{\&}\end{array}$

* Ajustado por: sexo, escolaridad materna (años), tipo de familia (nuclear / extendida) y lugar que ocupa el infante dentro de la familia (primero vs. segundo o más)

¥ Ajustado por: sexo del infante y escolaridad materna (años).

$\S$ Ajustado por: sexo del infante, tipo de familia (nuclear / extendida) y lugar que ocupa el infante dentro de la familia (primero vs. segundo o más)

\# Ajustado por: sexo, escolaridad materna (años) y lugar que ocupa el infante dentro de la familia (primero vs. segundo o más)

$\& p<0.05$ 


\section{Discusión}

En nuestro estudio no se encontró ninguna asociación del desarrollo motor con la escolaridad materna, el tipo de familia (nuclear o extensa) y si el niño es primogénito o no, sin embargo, estudios previos han identificado que la socialización, clase social, edad, escolaridad materna y/o medio ambiente en el que se desenvuelve el niño influyen en su motricidad total. ${ }^{8-10,12,14}$ Estas inconsistencias pueden ser debidas a que en nuestra población existe poca variabilidad en relación a escolaridad materna y nivel socioeconómico.

Nuestros resultados también sugieren que el género se asocia con el desempeño motor fino y grueso. Las niñas muestran una mayor facilidad para la realización de tareas de motricidad fina, mientras que los niños la tienen en la motricidad gruesa. Estos resultados concuerdan con lo reportado por otros autores, ${ }^{8,24,25}$ quienes encuentran una diferencia en el rendimiento motor entre niños y niñas y sugieren que esto puede ser el reflejo de la influencia de la escuela, la familia y los medios de comunicación para condicionar cierto tipo de conductas frente a otras de acuerdo con el género; por ejemplo, al promoverse los movimientos corporales bruscos en los niños, mientras que en las niñas se promueven la destreza manual y las coordinaciones finas; aunque también hay autores que afirman que estas diferencias de género son debidas a factores biológicos. ${ }^{26}$ Por otro lado, lo encontrado no concuerda con un estudio realizado por Ontiveros y cols., ${ }^{14}$ quienes no encontraron diferencias por género, aunque en ese estudio sólo se evaluó la motricidad gruesa.

De acuerdo con nuestros resultados, los niños reciben más estimulación en casa que las niñas; tienen más oportunidad de ser incluidos en las actividades diarias y eventos de la familia, además de tener un mayor acercamiento con la madre. Al respecto, Handal y cols., ${ }^{6}$ afirman que la variedad en las actividades madre-hijo favorece el desarrollo motor, cognitivo y social.

Los datos encontrados también indican que el acercamiento con el niño dentro del hogar y su estimulación influyen significativamente en su desarrollo motor; estudios previos han demostrado que la depresión materna se asocia negativamente con la estimulación en el hogar ${ }^{1}$ y que las diversas actitudes paternas tienen efectos importantes en la motricidad infantil. ${ }^{8}$ Tal es el caso del denominado temperamento apagado (madres con CI bajo, pobre estimulación en casa y ausencia paterna). ${ }^{27}$ Incluso eventos tales como el amamantamiento ${ }^{28}$ o peso bajo al nacer ${ }^{29}$ no son significativos frente a la calidad de estimulación en el hogar, siendo este último el factor asociado a la motricidad del niño. A diferencia de estos estudios, Ontiveros y cols. ${ }^{14}$ no encontraron asociación entre la estimulación total en el hogar y las conductas motoras gruesas evaluadas.

Es así que la estimulación que se le brinda al niño en el hogar contribuye con su desarrollo motor; sin embargo, particularmente los componentes de la estimulación medidos a través de la Escala HOME que se asocian con la motricidad gruesa son diferentes de los que se asocian con la motricidad fina.

Thelen $^{30}$ afirma que el desarrollo motor del niño no sólo depende de su estado orgánico, sino también de su historia de experiencias pasadas y de cómo fue la interacción con esa tarea en particular, además de ser de gran importancia las experiencias diarias que contribuyen en el aprendizaje de las habilidades motoras. En concordancia nuestros resultados, Thelen ${ }^{30}$ encuentra que el ambiente donde se desenvuelve el niño se asocia con su motricidad, aunque adicionalmente nuestro estudio también identifica ciertas características particulares de este ambiente familiar y su relación con ciertas características del desarrollo motor.

En relación con la motricidad gruesa, las conductas motrices que están particularmente asociadas a la estimulación en el hogar son el equilibrio y la locomoción. Se encontró que cuando la madre realiza conductas como dar libertad al niño para explorar su medio ambiente, vocalizar espontáneamente mientras está en casa cerca del niño y reforzar sus acciones positivas a través de hablarle, acariciarlo o teniendo un acercamiento físico con él favorece en el niño la habilidad para moverse de un lugar a otro caminando, corriendo y/o saltando. Asimismo se observó que cuando la madre no restringe y no castiga innecesariamente al niño, aceptando su presencia y sus conductas de exploración pero estableciendo límites, favorece la habilidad del niño para controlar su propio cuerpo en relación al centro de gravedad, mejorando su equilibrio y autocontrol corporal.

Por su parte, la motricidad fina que incluye actividades motoras de agarre e integración visomotora se asoció también con aspectos de la estimulación en el hogar. Así, cuando la madre realiza conductas donde vocaliza, refuerza y promueve la libertad del niño para explorar su medio ambiente, donde proporciona al niño juguetes adecuados a su edad que le ayudan a desarrollar la destreza motora fina a través de actividades lúdicas, al involucrarse activamente en el aprendizaje del niño y al proporcionar estimulación para incrementar la madurez conductual, incluyendo al niño en actividades diarias y manteniendo un contacto cercano con él, favorece la habilidad del niño para utilizar las manos y dedos en actividades que requieren de coordinación ojo-mano, como tomar objetos, construcción con cubos o copia de trazos. En este sentido, en Brasil, De Barros ${ }^{7}$ encontró que el desempeño motor fino se afecta negati- 
vamente por el uso de juguetes que no son adecuados a la edad de los niños, así como un espacio poco propicio para realizar actividades motoras. Asimismo, Lima ${ }^{31}$ afirma que cuando la interacción madre-hijo se incrementa en niños de un año de edad hay un aumento de la motricidad total en 8.2 puntos, usando la Escala de Desarrollo Infantil de Bayley, mientras que $\mathrm{Anme}^{32}$ en Japón encuentra que en niños de 1 año de edad la eficacia en el cuidado materno favoreció también el desempeño motor, y es la conducta de los padres hacia los hijos el factor asociado más importante.

En resumen, a diferencia de estos estudios este trabajo destaca particularmente los componentes de la motricidad que se ven favorecidos por la estimulación en el hogar, que son el equilibrio y la locomoción en la motricidad gruesa y el agarre e integración visomotora en la motricidad fina, y no sólo en la motricidad total descrita en otros trabajos. Los datos de otros estudios concuerdan con éste en uno de los aspectos de la estimulación en el hogar: la interacción madre-hijo como algo esencial y particular de la estimulación que favorece la motricidad; sin embargo, este estudio aporta adicionalmente que es también la libertad que se le da al niño para explorar su medio ambiente, reforzar sus acciones positivas a través de hablarle y no restringir ni castigar innecesariamente al niño, aceptando su presencia y sus conductas de exploración pero proporcionando límites, lo que está particularmente asociado a las habilidades motrices.

Los resultados encontrados dejan ver la necesidad de establecer programas de educación a padres que fomenten la estimulación de los niños en casa para favorecer su desarrollo integral, además de dirigir el trabajo no sólo a ciertos grupos sino a la población en general.

En algunos países de América Latina los programas educativos brindan asesoría a padres, madres y personas que participan en el cuidado de niños y niñas en edades tempranas en comunidades rurales e indígenas con el fin de enriquecer las prácticas de crianza; asimismo, se establecen centros de cuidado infantil encaminados al cuidado y estimulación de niños de madres trabajadoras; algunos otros ofrecen estimulación dirigida a niños con necesidades especiales, de tal forma que la población general que no entra en ninguno de estos grupos no recibe ningún tipo de información y capacitación para mejorar la crianza de los niños en su medio familiar. En las escuelas hay más contacto con los padres de familia y las pláticas para padres son la mejor forma de coordinar un trabajo conjunto entre ésta y la casa; sin embargo, este estudio revela la importancia de hacerlo en los primeros años de vida del infante, antes de su ingreso al nivel preescolar. Es por ello que los programas de estimulación, además de ser dirigidos a la población en general, deberían ser impartidos en edades tempranas, fomentando así un desarrollo integral del niño.

En conclusión, es importante que los padres puedan ser asesorados en cuanto a la estimulación que pueden ofrecer a sus hijos dentro del hogar, pues más de $80 \%$ de los niños son cuidados en casa en los primeros tres años de vida y este puede ser un ambiente favorable o desfavorable para su óptimo desarrollo. Se hace énfasis en la necesidad de establecer un vínculo más cercano con los niños y de incrementar la comunicación y convivencia familiar, estableciendo reglas claras; particularmente favorecer en los niños actividades motoras finas como colorear, recortar, ensartar y abotonar, y en las niñas actividades motoras gruesas utilizando pelotas, cuerdas y aros, y movimientos de carrera y salto. Todo lo anterior puede ser difundido en los centros de salud de la comunidad donde, además de las consultas médicas necesarias para el bienestar físico, se pueda sensibilizar a los padres hacia la relevancia de hacer estas actividades en casa. Aunado a esto, es importante señalar la necesidad de establecer estos programas desde las primeras consultas de revisión médica a las que asiste el niño que acaba de nacer.

Una limitante del estudio es que no se tomaron en cuenta otras variables como tipo de crianza o depresión materna que pueden también contribuir en las habilidades motoras evaluadas, por lo que se sugiere su inclusión para estudios futuros. Asimismo, no se hizo una validación para la Escala HOME en este estudio; sin embargo, los datos obtenidos se encuentran dentro de los rangos propuestos por la misma.

\section{Agradecimientos}

Este trabajo fue financiado por el Consejo Nacional de Ciencia y Tecnología de México/CONACyT (41708, 31034-M, 13915) y por Fogarty a tráves del Mount Sinai School of Medicine International Training and Research in Environmental and Occupational Health Program (D43TW00640).

\section{Referencias}

I. Black MM, Baqui AH, Zaman K, McNary SW, Le K,Arifeen SE, et al. Depressive symptoms among rural Bangladeshi mothers: implications for infant development. J Child Psychol Psychiatry 2007;48:764-772. 2. Kolobe TH, Bulanda M, Susman L. Predicting motor outcome at preschool age for infants tested at 7,30,60, and 90 days after term age using the Test of Infant Motor Performance. Phys Ther 2004;84: I I 44- I I 56. 3. Burchinal MR, Roberts JE, Riggin R Jr, Zeisel SA, Neebe E, Bryant D. Relating quality of center-based child care to early cognitive and language development longitudinally. Child Dev 2000;71:339-357. 
4. Lejarraga H, Pascucci M, Krupitzky S, Kelmansky D, Bianco A, Martínez E, et al. Psychomotor development in Argentinean children aged 0-5 years. Paediatr Perinat Epidemiol 2002;16:47-60.

5. Papalia D. Psicología del desarrollo. Santa Fé de Bogotá, Colombia: Mc.Graw-Hill, 1992.

6. Handal AJ, Lozoff B, Breilh J, Harlow SD. Sociodemographic and nutritional correlates of neurobehavioral development: a study of young children in a rural region of Ecuador. Rev Panam Salud Publica 2007;21:292-300.

7. De Barros KM, Fragoso AG, de Oliveira AL, Cabral-Filho JE, de Castro RM. Do environmental influences alter motor abilities acquisition? A comparison among children from day-care centers and private schools. Arq Neuropsiquiatr 2003;61: 170-175.

8. Ruiz L. Desarrollo motor y actividades físicas. Madrid España: Gymnos, 1987.

9. Ponjaert-Kristoffersen I,Tjus T, Nekkebroeck J, Squires J,Verté D, Heimann M, et al. Psychological follow-up study of 5-year-old ICSI children. Hum Reprod 2004;19:2791-2797

10. Soler K, Rivera I, Figueroa M, Sánchez L, Sánchez M. Relación entre las características del ambiente psicosocial en el hogar y el desarrollo psicomotor en el niño menor a 36 meses de edad. Bol Med Hosp Infant Mex 2007; 64:273-287.

I I. Mendelsohn A, Mogilner L, Dreyer B, Forman J,Weinstein S, Broderick $M$, et al.The impact of a clinic-based literacy intervention on language development in inner-city preschool children. Pediatrics 200I;107(I):I30-I34. 12. Torralva T, Cugnasco I, Manso M, Sauton F, Ferrero M, O'Donnell A, et al. Desarrollo mental y motor en los primeros años de vida: su relación con la estimulación ambiental y el nivel socio-económico. Arch Argent Pediatr 1999;97:306-316.

13. INEGI/IMSS. Encuesta Nacional de Empleo y Seguridad Social 2004. [Consultado septiembre 13 2009]. Disponible en: www.inegi.gob.mx 14. Ontiveros E, Cravioto J, Sánchez C, Barragán G. Evaluación del desarrollo motor en función de género, estimulación disponible en el hogar y nivel socioeconómico en niños de 0 a 3 años de edad del área rural. Bol Med Hosp Infant Mex 2000;57;3 I I-3 I9.

15. Torres-Sanchez L, Rothenberg SJ, Schnaas L, Cebrian ME, Osorio E, Hernandez M, et al. In utero p,p'-DDE exposure and infant neurodevelopment: a perinatal cohort in Mexico. Environ Health Perspect 2007; I1 5:435-439.

16. Folio MR, Fewell RR. Peabody Developmental Motor Scales (PDMS-2). Second Edition. Austin Texas: Pro-ed Inc., 2000

17. Caldwell BM, Bradley R. Home Observation for Measurement of the Environment. Littlerock AR: University of Arkansas, 1984.
18. Riou E, Chosh S, Francoeur E, Shevell M. Global development delay and its relationship to cognitive skills. Developmental Medicine and Child Neurology 2009;5 I:(3)600-606.

19. CoolsN, De Martelaer K, Samaey C,Andries C. Movement skill assessment of typically developing preschool children:A review of seven movement skill assessment tools. Journal of sports science and medicine 2008:8:154-168

20. Mayrand L, Mazer B, Menard S, Chilingaryan G. Screening for motor deficits using the Pediatric Evaluation of Disability Inventory(PED) in children with language impairment. Dev Neurorehabil 2009;12(3):।39-145. 21. Chi-Wen Ch, Bond T. Measurement properties of fine motor scale of Peabody development motor scales second edition. Am J Phys Med Rehabil 2009;88(5):376-386.

22. Provost B, Heimerl S, Lopez B. Levels of gross and fine motor development in young children with autism spectrum disorder. Phys Occup Ther Pediatr 2007;27(3):21-36.

23.Wechsler D.WAIS-Español Escala de Inteligencia para Adultos. México DF: Manual Moderno, 198I.

24. Moraleda M. Psicología del desarrollo. México DF:Alfaomega, 1999 25. Gillette EA, Meza MM, Aguilar MG, Soto AD, García IE.An antropological approach to the evaluation of preschool children exposed to pesticides in Mexico. Environ Health Perspect 1998;106:347-353. 26. Slee P. Child, adolescent and family development. Second edition. United Kingdom: Cambridge University Press, 2002:133

27. De Andraca I, Pino P, de la Parra A, Rivera F. Factores de riesgo para el desarrollo psicomotor en lactantes nacidos en óptimas condiciones biológicas. Rev Saude Publica 1998; 32: 479-487.

28. Eickmann S, Cabral-de Lira P, De Carvalho M, Bechara S, Pérez M, et al. Breast feeding and mental and motor development at 12 months in a low-income population in northeast Brazil. Paediatr Perinat Epidemiol 2007;21:129-137.

29. Emond A, Lira P, Lima M, Grantham S, Ashworth A. Development and behavior of low-birthweight term infants at 8 years in northeast Brazil:A longitudinal study.Acta Pediatrica 2006;95:I249-I257.

30. Thelen E. Motor development as foundation and future of developmental psychology. Int J Behav Dev 2000; 24(4): 385-397. 3I. Lima MC, Eickmann SH, Lima ACV, Guerra MQ, Lira PIC, Hunttly SRA, et al. Determinants of mental and motor development at 12 months in a low income population: a cohort study in northeast Brazil.Acta Pediatr 2004;93: 969-975.

32.Anme T, Uma AS. Implications of Japan's center-based night care a I year follow-up. Early Childhood Educ J 2007;35:293-299. 